FERMILAB-Conf-91/153

\title{
Design and Multiparticle Simulation of the Half Integer Slow Extraction System for the Main Injector
}

\author{
D. Trbojevic and M. Harrison
}

Fermi National Accelerator Laboratory

P.O. Box 500, Batavia, Illinois 60510

May 1991

* Presented at the 14th Biennial IEEE Particle Accelerator Conference, San Francisco, CA, May 6-9, 1991. 


\section{Disclaimer}

This report was prepared as an account of work sponsored by an agency of the United States Government. Neither the United States Government nor any agency thereof, nor any of their employees, makes any warranty, express or implied, or assumes any legal liability or responsibility for the accuracy, completeness, or usefullness of any information, apparatus, product, or process disclosed, or represents that its use would not infringe privately owned rights. Reference herein to any specific commercial product, process, or service by trade name, trademark, manufacturer, or otherwise, does not necessarily constitute or imply its endorsement, recommendation, or favoring by the United States Government or any agency thereof. The views and opinions of authors expressed herein do not necessarily state or reflect those of the United States Government or any agency thereof. 


\title{
Design and Multiparticle Simulation of the Half Integer Slow Extraction System for the Main Injector
}

\author{
D. Trbojevic and M. Harrison \\ Fermi National Accelerator Laboratory* \\ P.O. Box 500, Batavia, Illinois 60510
}

\begin{abstract}
One of the roles of the new Main Injector ring, in the secund phase of the Fermilab upgrade, is to deliver all year round 1. he slow extracted $120 \mathrm{GeV}$ test beams. The half-integer slow ixtraction system design and results from a Monte-Carlo simufition of fast spill are presented. The simulation was performed wh a computer tracking program based on the TEVLAT pro"rritu witlt a large number of particles (up to 1000). Particle liacking included the systematic errors produced by the magie: ic multipoles within the dipoles and quadrupoles as well as rianclom multipole errors.
\end{abstract}

\section{Introduction}

The: Main Injector will replace the existing Main Ring in the licrmilab Accelerator Complex and allow significant enhancements to both the Fermilab collider and fixed target programs. Vumber of protons delivered to the antiproton production taris: or total number of protons delivered to the Tevatron will lu: lwo to thrce times higher than the present one. In addilion the Main Injector will provide continuous $120 \mathrm{GeV}$ beam w the experimental area during the collider operation, a cafiability which does not presently exist in the Main Ring [1]. f'lo: Main Injector will be built from newly constructed dipole minucts. In this report we present a design system for the inlf-integer resonant extraction for the Fermilab Main Injec10r. One of the major intentions of this project is to reconfirm the quality of the magnetic field in the Main Injector dipole and quadrupole magnets at $120 \mathrm{GeV}$ operation. The beam ilze at $120 \mathrm{GeV}$ is too small (for the normalized emittance of ' $=40 \pi \mathrm{mm} m \mathrm{mrad}$ the beam $\sigma_{\max } \approx 1.7 \mathrm{~mm}$ ) to cause any incerns about the aperture. The particle trajectories during 1 hi: slow extraction process attain large betatron amplitudes. Mre ficld quality always deteriorates away from the axis. At l.igh values of the magnetic field slow extraction represents the major aperture probe and sets the limits on the magnetic field fritlity. In the simulation process different quality of the maglat ir field in the dipoles and in the quadrupoles were examined. I. l. he first part of the report a short introduction into the half litrger resollant extraction is presented with the positions and rules of the quadrupole and octupole magnets. A modeling of Whe half-integer resonant extraction with a computer program hased on the TEVLAT [2] is shown in part III. The magnetic lield errors in the dipoles and the quadrupoles were included in llic simulation and the results of a Monte-Carlo simulation are presented.

- Operated by the Universities Research Association under conarict with the U. S. Department of Energy

\section{Half Integer Extraction in the Main Injector}

The Main Injector lattice used in the half integer extraction system is based on a FODO cell structure with a $90^{\circ}$ basic cell [1]. This lattice, as reported in the conceptual design, had both the horizontal and vertical tunes close to a value of 22.4 , and natural chromaticities of $\xi_{x}=-28.02$ and $\xi_{y}=-28.35$. There were a total of 300 hundred dipoles in the ring. The maxima and minima of the betatron functions in the FODO cells wero $\beta_{\max } \approx 58$ meters and $\beta_{\min } \approx 11$ meters. The maximum momentum is $150 \mathrm{GeV} / \mathrm{c}$ while the slow extraction is performad at $120 \mathrm{GeV} / \mathrm{c}$. This choice is related to the dipole's magnetic field properties at the high field values-multipole content (the momentum of $150 \mathrm{GeV} / \mathrm{c}$ requires the magnetic field of 17.26 kG which is close to saturation). The slow extraction electrostatic and magnetic septa are located at the fast, one-pulse extraction straight section. The magnetic septa-Lambertson magnets will be common elements for both fast and slow extrac. tion processes. The fast kicker magnets, used for the one pulse fast-extraction, were located in the design report upstream of the Lambertson magnets with a betatron phase difference of $90^{\circ}$. During the slow extraction process the beam is horizontally deflected into the field region of the Lambertson magnet where it is bent upward.

The choice of the half integer slow extraction for the Fermilab Main Injector came mostly from the experience during operations in both Fermilab accelerators-the Main Ring and the Tevatron. Although both the third and half integer resonant extraction processes were designed in detail during the Tevatron design the half integer was chosen for operation [3]. The half integer resonance is a linear resonance and the beam is cither stable or unstable. The resonant extraction starts with a change of the tune toward the half integer value $\left(\nu_{x}=22.5\right)$. The zero-harmonic octupole introduces a tune shift $\Delta \nu_{x} \propto x^{2}$. A combination of the linear-quadrupole and nonlinear- octupole elements creates a stable region in the phase space where a particle oscillates between the "fixed" points. The amplitusle of oscillation increases from turn to turn. The motion of particles is presented in figure 1 [4]. The central stable phase space is reduced in size to be too small to contain the heam. Particles then follow the trajectories of the unstable phase space increasing their amplitudes of motion. The simulation of the slow extraction process included only a pair of quadrupoles with opposite polarities and a zero-harmonic octupole at the same location as the slow extraction focussing quadrupole. The slow extraction focussing quadrupole was located upstream of the electrostatic septum with a betatron phise difference of $\sim 90^{\circ}$ degrees with respect to the septum. The other, defocusing slow 
Etraction guadrupole, was located at the opposite side of the ring.

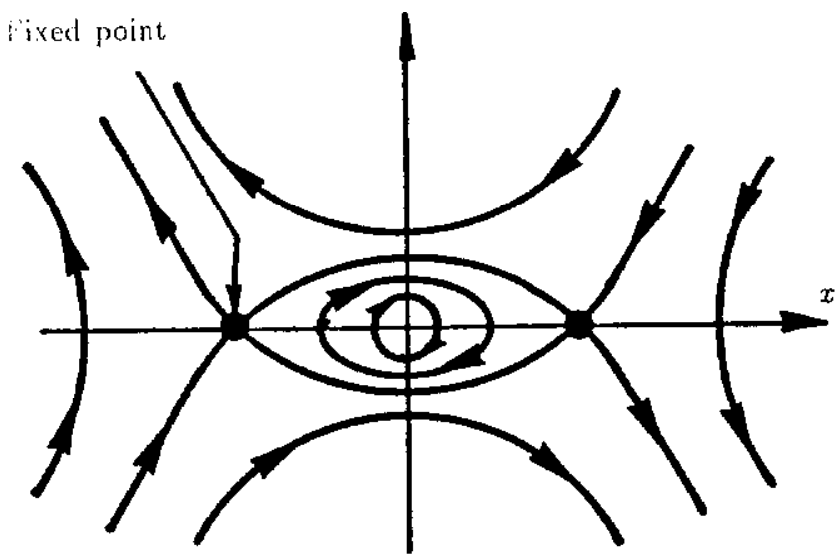

ligure 1. The phase space trajectories during the half integer ronant slow extraction.

The position of the electrostatic septum was selected at the avalable drift space upstream of the Lambertson magnet with a betatron plase difference of $75.66^{\circ}$ degrees. It is always bett.cr to position the electrostatic septum downstream of the fast kicker magnets due to unavoidable small beam losses at the wire itray of the septum. The beam loss will not induce radioactive damage to the kicker magnet. The length of the electrostatic ceptum was 3.6 meters. The septum high voltage was $54.4 \mathrm{kV}$ across a $14 \mathrm{~mm}$ gap between the electrode and the wire array. This kick was strong enough to establish a $6 \mathrm{~mm}$ distance for 1.he extracted beam at the magnetic septum. The magnetic wpl.11m. Lambertson magnet has a magnetic field-free region whe the beam circulates through the center of the aperture. The beam is bent in the vertical plane within the extraction diannel. The smallest separation between the field free region and the extraction channel is $2 \mathrm{~mm}$. The separation wall of the vacuum chamber in the field free region has two sides with an ingle of $45^{\circ}$ degrees.

\section{Computer Simulation}

The slow extraction should provide a smooth constant rate of extracted beam throughout the cycle. Particles in the beam have unavoidable tune spread which arises from the final beam momentum spread: the tune shift due to chromatic effects and pace clarge tune-shift which is intensity dependent. The tune spread also comes from the nonlinear elements: the guide field nonlinearities, sextupoles or other nonlinear elements which inHuce betatron amplitude tune shift. The spill rate is usually divided into two kinds: a slow spill - where the length of the spill is of the order of more than one second and a fast spill where the length of the spill is of the order of 200 turns (one urn $\simeq 10 \mu \mathrm{sec}$ ). The time scale of the fast spill is small enough l liat a computer simulation of the complete process (with 1000 inrus) is possible without any approximation within reasonible computer time. The computing was performed with the workstation SUN SPARC-1. The code in simulation, devel1) ped in Fermilab [2], is a kick code where the particie ensemble is propagated from element to element. The multipole field of the dipoles is presented with kicks of thin elements in the middle of the dipole. The simulation of the extraction process was performed only after the length of the ring, the natural chromaticities, the betatron functions, and the tunes obtained by one particle tracking showed complete agreement with results obtained with the SYNCH computer program. The particles' (400-1000 particles) initial positions, slopes of both coordinates, and momentum offsets, were chosen randomly. When the slow extraction process was simulated the gradients of the main quadrupoles in the lattice were first set to produce the horizontal tune of 22.485 with the vertical tune at a previous value of 22.4. The chromaticity sextupoles changed the chromaticities to values $\sim+3$. The slow extraction quadrupoles and zero-harmonic octupoles were tuned to produce a stable phase space where an ensemble of particles oscillates with well defined stable points, as presented in fig. 1. The slow extraction quadrupoles could follow either a half sinusoidal or a linear ramp pulse above a d.c. value. The extraction of particles occurred when the pulsed part of the slow extraction quadrupoles was turned on. The electrostatic wire array was placed at 17 $\mathrm{mm}$ away from the center of the horizontal aperture. When a particle reached this distance it would undergo a horizontal kick of $0.12 \mathrm{mrad}$. If during this motion in a horizontal space a particle hit the wire (effective thickness of $0.1 \mathrm{~mm}$ ) it was considered lost. This was used as an efficiency measurement and at the same time this was a way to determine if the step size in process was correctly defined. During a design procedure there is always a compromise between the efficiency which can be reached and the shape of the phase space of the extracted particles. The further the position of the septum array from the aperture center is the smaller losses are, but the worse magnetic field in the dipoles is. The maximum horizontal offset through the dipoles, in the whole Main Injector ring and before particles reached the septum array in the ring, was $44.9 \mathrm{~mm}$. Because the electrostatic septum was placed close to the extraction Lambertson septum the extracted particles travel through a short part of the ring. The maximum horizontal offset in the magnets reached by the extracted particles was $25.4 \mathrm{~mm}$. At the time of the tracking studies the new Main Injector magnet had not been built yet and the magnetic field quality was not known. The magnetic multipoles in the dipole field $B(x)$ at offset $x$ are defined as $B(x)=B_{0}\left[1+b_{2} / 2 x^{2}+b_{4} / 12 x^{4}+\cdots\right]$, and $B_{0}$ is the vertical field at the center. The Main Injector will replace the present Main Ring in Fermilab. The multipole content of the two kinds of the Main Ring dipoles (B1 and $\mathrm{B} 2$ dipoles) is known and has been measured. In the simulations a set of multipoles from the B2 Main Ring dipoles, which should resemble the Main Injector dipoles, were used. Because the Main Ring dipoles saturation occurs at higher current values (magnets were designed for the $400 \mathrm{GeV}$ machine) the values of all multipoles were doubled. At Fermilab values of the multipoles in the magnetic field are defined as the relationship between the field produced by the multipole and the main dipole field $b_{0}$ at the distance of one inch multiplied with $10^{4}$. The average values of the measured (at the current of $1700 \mathrm{~A}$ ) normal multipoles in the Main Ring dipoles are presented in Table 1. The multipole expansion was cut at the eleventh pole. The simulation was performed with twice higher multipoles than those presented in table 1 . The limits in the values of multipoles were set by the standard deviations obtained in magnetic measurements (in the table 1 "sdev"). 
Table 1

Normal Multipoles $\times 10^{4}$

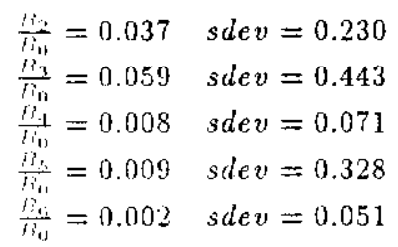

Skew Multipoles $\times 10^{4}$

$$
\begin{aligned}
& \frac{B_{2}}{B_{0}}=3.910 \quad \text { sdev }=0.391 \\
& \frac{B_{3}}{B_{0}}=0.049 \quad \text { sdev }=0.197 \\
& \frac{B_{4}}{B_{0}}=0.321 \quad \text { sdev }=0.221 \\
& \frac{B_{s}}{B_{0}}=-0.026 \quad \text { sdev }=0.046 \\
& \frac{B_{s}}{B_{0}}=0.092 \quad \text { sdev }=0.161
\end{aligned}
$$

'The distributions of an ensemble of 400 particles in the phase inace at the electrostatic and magnetic septum, during the simwhtion, are presented in figures 2 and 3, respectively.

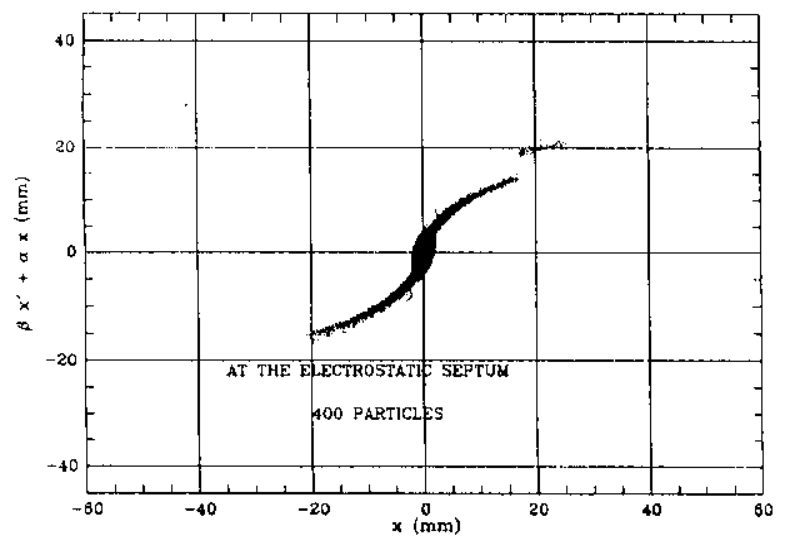

Pigure 2. Slow extraction simulation during the fast spill. The distribution of 400 particles in the phase space at the electrostatic septum ( 540 turns).

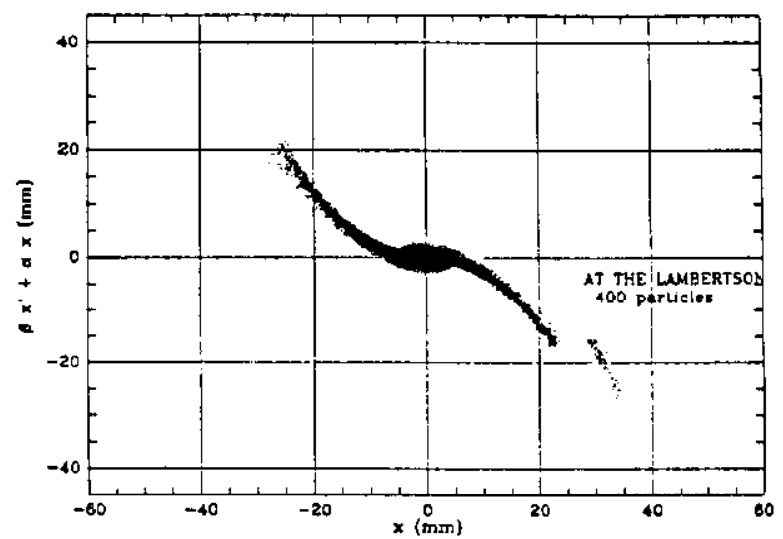

Figure 3. Slow extraction simulation during the fast spill. The distribution of 400 particles in the phase space at the Lambertson magnel (540 turns).

Nost of the quadrupoles in the Main Injector will be the rcissed Main Ring quadrupoles (according to the design report [1]). At the high field the octupole harmonic in the Main Ring quadrupoles had been measured to be 6 Fermilab units with respect to the field of the quadrupoles at 1 inch $\left(B_{4} / B_{2}=8.0 \times 10^{-4}\right)$. Another set of simulation runs with the mcasured value of the octupole multipole within the quadrupole magnets was performed. The influence of the octupole har" monic from the quadrupoles on the particle distribution in the Hhise space showed that the strength of the zero-harmonic ocunole needed to be lowered. The error in quadrupole misalign- ment, always present in the real machine, produces a natural half integer stop band and propagates at twice the tune (45th harmonic). The slow extraction quadrupoles will be used at the same time to correct the natural stop band of the $45 \mathrm{th}$ harmonic. In the simulation a presence of the natural $45 \mathrm{tl}$ harmonic was not taken into account. The strength of the slow extraction quadrupoles at the best efficiency with a particlo loss of less than $1 \%$ was $5.4 k \mathrm{Gm} / \mathrm{m}$ (at $120 \mathrm{GeV} / \mathrm{c}$ ) while the strength of the octupole was $2.7 \times 10^{4} \mathrm{kGm} / \mathrm{m}^{3}$. The octupole strength is a strength of a single octupole. In the Main Injector zero-harmonic octupoles will be distributed around the ring.

\section{Concluding Remarks}

One of the roles of the future Main Injector will be to provide slow extracted beams up to $3 \times 10^{13}$ protons to the experimental areas during the collider runs for use in high sensitivity $\mathrm{K}$ decay and neutrino experiments and for the test beams. The design of the half-integer slow extraction system and the multiparticle simulation of the extraction process has been presented. The simulation has shown that it is possible to design an efficient half-integer slow extraction system with the expected field quality of the Main Injector magnets. The results of the simulation showed that the new Main Injector magnets should have better multipole content than twice the size of the multipoles within the B2 Main Ring magnet at $120 \mathrm{GeV}$. The simulation also showed that the distortion of the extracted particles phase space is acceptable even with the maxima in the horizontal offsets through the dipoles during the last extraction turns of the order of $40 \mathrm{~mm}$. The available aperture fulfills conditions required for the slow extraction beam trajectories. The elements of the slow extraction system fit well within the available drift space of the lattice presented in the design report [1]. For the slow extraction quadrupoles it is possible to use the existing Main Ring small harmonic correction quadrupoles $(20 \mathrm{~cm}$ long with an integrated strength of $0.255 \mathrm{kGm} / \mathrm{m} / \mathrm{A}$ [5]). The strength of the zero-harmonic octupoles would require 50 octupoles of the same strength as the Main Ring chromaticity octupoles with a current through the coils of $20 \mathrm{Amp}$ (the strength of the Main Ring harmonic octupoles is $\left.b_{3} L_{e f f}=27.92 \mathrm{kGm} / \mathrm{m}^{3} / A[5]\right)$.

\section{References}

[1] “The Main Injector Lattice, ${ }^{n}$ Conceptual Design ReportFermilab Upgrade:Main Injector, Internal Report, Fermilab, Batavia, March 1989, pp. 8-16.

[2] Norman Gelfand, "Fermilab Tracking Codes,"Fermilab, Batavia, Illinois, Internal Memo, May 14, 1990, pp. 1-29.

[3] D.A.Edwards, "Comparison of Half Integer and Third Integer Extraction for the Energy Doubler," Fermilab, Batavia, Mlinois, Internal Report: TM-812, December 1978, pp 1.29.

[4] M. Harrison, "Resonant Extraction at the Tevatron,"Nev York: American Institute of Physics, 1989, AIP Conference Proceedings 184 - Physics of Particle Accelerators, Fermilab Summer School, 1987, pp. 2009-2032.

[5] D. Trbojevic, "Magnetic Measurements of the Correction and Adjustment Magnets of the Main Ring," Fermilab, Batavia, Mlinois, TM-1412, July 1986, pp. 1-38. 